\title{
Zur historisch-geographischen Epidemiologie der Poliomyelitis
}

\author{
Helmut Knolle
}

\section{SuMmary}

Poliomyelitis was considered a rare disease before it terrified Europe and North America with large-scale epidemics during the first half of the 20th century. In Africa and Asia the number of reported cases increased remarkably only after World War II. A theory which is widely accepted today assumes that infection with poliovirus 1, 2 and 3 has always been globally endemic, but that the proportion of cases with residual paralysis has increased only since 1900 as a consequence of the rise of the mean age at infection. Sabin, however, initially was convinced that virus strains with enhanced neurotropism had caused the dramatic increase in paralytic poliomyelitis. Epidemic outbreaks in anglo-american troops in Malta and in the Far East during the war played a crucial role in the discussion. Later, also Sabin sustained the theory mentioned first, which gradually assumed the position of a dogma. The present paper deals with the question of how this dogma became dominant, in spite of the weakness of its epidemiological and virological foundation.

\section{Zusammenfassung}

Die spinale Kinderlähmung ( Poliomyelitis) galt als seltene Krankheit, ehe sie in der ersten Hälfte des 20. Jahrhunderts Europa und Nordamerika mit Epidemien von zunehmender Heftigkeit heimsuchte. In Afrika und Asien nahm die Zahl der Meldungen erst nach dem 2. Weltkrieg sehr stark zu. Eine heute allgemein akzeptierte Theorie nimmt an, dass die Infektion mit Poliovirus 1, 2 und 3 früher weltweit endemisch gewesen sei, dass aber der Anteil der Infektionen mit bleibenden Lähmungen erst seit 1900 zugenommen habe, und zwar als Folge eines Anstiegs des mittleren Infektionsalters. Sabin, der den oralen Impfstoff entwickelte, war jedoch anfänglich davon überzeugt, dass Virusstämme mit 
erhöhter Neurotropie für die Zunahme der Erkrankungen verantwortlich waren. Epidemische Ausbrüche unter anglo-amerikanischen Soldaten auf der Insel Malta und im Fernen Osten während des Krieges spielten eine Schlüsselrolle in der Diskussion. Später propagierte auch Sabin die zuerst genannte Theorie, die allmählich die Rolle eines Dogmas übernahm. Der folgende Beitrag geht der Frage nach, wie dies Dogma zur Herrschaft kommen konnte, obwohl es epidemiologisch und virologisch kaum begründet ist.

Die Weltgesundheitsorganisation (WHO) hat sich die globale Ausrottung der Poliomyelitis (spinale Kinderlähmung) bis zum Jahr 2000 zum Ziel gesetzt. Dies ist Anlass genug, um sich noch einmal die Epidemiologie und Geschichte dieser Krankheit, die in der Dritten Welt heute noch viele Opfer fordert, in Erinnerung zu rufen. Es wird sich zeigen, dass die Frage, woher die Kinderlähmung kam und warum sie überhaupt zu einem globalen Problem wurde, immer noch ungeklärt ist. Die These, dass die Poliomyelitis ursprünglich eine auf Europa und Nordamerika beschränkte Krankheit war, die sich erst in der Dekade nach dem 2. Weltkrieg in Asien und Afrika ausgebreitet hat, ist in den 50er Jahren verdrängt worden, obwohl die Gegenargumente kaum überzeugen können. Seitdem herrscht die Meinung, dass die Poliomyelitis schon vor dem 2. Weltkrieg pandemisch gewesen sei. Im folgenden soll nach einer Einführung in das Problem die in den 40er und 50er Jahren geführte Diskussion kritisch dargestellt werden.

\section{Historischer Rückblick}

Als Jakob Heine 1860 seine Monographie «Spinale Kinderlähmung» ${ }^{1}$ publizierte, handelte es sich noch um eine relativ seltene Krankheit, die gelegentlich bei Kindern beobachtet wurde. Dass die Kinderlähmung infektiös sein könnte, kam Heine nicht in den Sinn, und als 21 Jahre später das in seiner Vollständigkeit nie wieder erreichte «Handbuch der historisch-geographischen Pathologie» von August Hirsch erschien, war die Kinderlähmung darin nicht erwähnt.

Erst um die Jahrhundertwende wurde die Krankheit einer breiteren Öffentlichkeit bekannt, und in den folgenden Jahrzehnten wurden die USA und Schweden, später auch die meisten west- und mitteleuropäischen Länder von schweren Epidemien heimgesucht. Auch Süd- und Osteuropa, Australien und Neuseeland blieben nicht verschont. Trotz intensivster Anstren- 
gungen in hygienischer Hinsicht nahm das Ausmass der Epidemien noch zu, bis die Entwicklung von Impfstoffen (Salk 1953, Sabin 1957) die Gefahr, zumindest in den Industrieländern, bannen konnte.

In Asien und Afrika ist bis zum 2. Weltkrieg über Kinderlähmung nur ganz vereinzelt berichtet worden. Dies kann wohl nicht überall auf Mängel der Diagnose und der Meldesysteme zurückgeführt werden. Sabin bemerkt zur Situation auf der Philippineninsel Leyte, die damals knapp 1 Million Einwohner hatte, vor der amerikanischen Invasion im Jahre 1944: «A careful investigation revealed no recent cases of poliomyelitis among the natives on Leyte. The last definite case of poliomyelitis reported from Leyte had been in 1938, and the civilian health records of the area were found to be remarkably complete because the Japanese authorities required a daily report on infectious diseases» ${ }^{2}$. Die Insel Leyte wird uns noch eingehend beschäftigen.

In der Dekade nach dem 2. Weltkrieg ist die Zahl der gemeldeten Fälle in Asien und Afrika in die Höhe geschnellt. In der belgischen Kolonie Kongo schwankte die Zahl vor 1950 um 90 und stieg dann auf 1009 (1951), und in Angola kletterte sie im gleichen Zeitraum von 15 auf 773. In Bombay ereignete sich 1949 die erste bekannte Epidemie, mit 389 Krankheits- und 65 Todesfällen. In Israel wurden 19501600 Fälle gemeldet, davon 154 mit tödlichem Ausgang. Nur 1\% der Fälle betrafen die arabische Minderheit, die damals etwa $20 \%$ der Bevölkerung bildete ${ }^{3}$.

Heute sind die Länder im tropischen Afrika am stärksten betroffen. In Ghana erkrankten zwischen 1970 und 1980 jährlich 31, an der Elfenbeinküste sogar 34 von 100000 Personen ${ }^{4}$. Obwohl schon in vielen Ländern mit gutem Erfolg geimpft wird, schätzt man die Zahl der Fälle mit Lähmungserscheinungen weltweit auf 250000 pro Jahr $^{5}$.

Die genannten Zahlen beziehen sich jeweils auf die Wohnbevölkerung der entsprechenden Länder. Das Bild wäre nicht vollständig, wenn wir nicht die Polio-Epidemien, die während des 2 . Weltkrieges bei anglo-amerikanischen Truppen in Asien und Afrika ausbrachen, erwähnen würden. Britische Truppen auf Malta und in Ägypten waren anscheinend die ersten, die mit dem Problem konfrontiert waren. Als im Herbst 1944 amerikanische Truppen in einer Stärke von 300000 Mann auf den Philippinen landeten, erkrankten bis zum Jahresende 39 Soldaten an Poliomyelitis, und im nächsten Jahr wurden bei den US-Truppen auf den Philippinen 246 Fälle registriert ${ }^{6}$. Auch in einer amerikanischen Division, die 1947 in China stationiert war, kam es zu einer Polio-Epidemie ${ }^{7}$. 


\section{2. Übertragungswege und Krankheitsverläufe}

Das Auftauchen der Kinderlähmung zuerst in den USA und in Europa und ihre anscheinend erst ein halbes Jahrhundert später erfolgte Verbreitung über die ganze Erde widerspricht der Regel, dass Infektionskrankheiten eher in armen als in reichen Ländern zu Hause sind. Allgemeine Regeln oder Gesetze sind hier wenig verlässlich, denn jede Infektionskrankheit hat spezifische Eigenschaften, so dass die Gültigkeit von allgemeinen Gesetzen in jedem besonderen Fall erst noch überprüft werden muss. Deshalb sollen hier zunächst die wichtigsten Tatsachen über Transmission, Pathologie und Immunologie der Poliomyelitis in Erinnerung gerufen werden.

Die Infektion mit Polioviren, den Erregern der Poliomyelitis, erfolgt durch den Mund. Danach vermehren sich die Viren im Magen-Darm-Trakt und gelangen mit dem Stuhl wieder in die Umwelt. Diese Phase kann bis zu 4 Monaten dauern. Die ersten Massnahmen der Prävention bestanden folglich darin, den direkten oder indirekten Kontakt (Kontamination der Nahrung durch Fliegen) mit menschlichen Fäkalien zu vermeiden. Aber trotz guter sanitärer Einrichtungen und persönlicher Hygiene kam es immer wieder zu Epidemien, denn wahrscheinlich war auch das Trinkwasser verseucht ${ }^{8}$. Jedenfalls sind die üblichen Methoden der Desinfektion unwirksam gegen Polioviren.

Das Eigenartige ist nun, dass die meisten Infektionen - wahrscheinlich mehr als 99\% - ohne Symptome verlaufen. Die restlichen 1\% zeigen ein weites Spektrum von Manifestationen, das von leichtem Fieber über vorübergehende oder dauernde Lähmungen bis zu tödlichem Ausgang reicht. $\mathrm{Zu}$ schweren Verläufen kommt es nur dann, wenn die Viren vom MagenDarm-Trakt auf dem Blutweg und über periphere Nervenbahnen zum Rükkenmark vordringen. Personen, die eine Infektion überstanden haben, sind für den Rest ihres Lebens immun. Deshalb waren die Erkrankungen eine Zeit lang auf das Kindesalter beschränkt. Durch die nicht ganz wirkungslosen Präventionsbemühungen wurde aber das mittlere Infektionsalter zunehmend angehoben.

\section{Zwei Theorien}

Es gibt im wesentlichen zwei sich widersprechende Theorien, die den Anspruch erheben, die geschilderten Vorgänge erklären zu können. Die eine, die 
Mutationstheorie, ist eine naheliegende Anwendung evolutionistischer Konzepte, wonach veränderte Umweltbedingungen in den Industrieländern Veränderungen im Genpool der Polioviren hervorgerufen haben. Die andere, die Theorie der Altersabhängigkeit, hält sich im Rahmen der herkömmlichen epidemiologischen Denkweise, für die Viren und Bakterien keiner historischen Wandlung unterworfen sind.

Die Mutationstheorie geht von den folgenden Überlegungen aus. Die Erreger der Poliomyelitis sind Viren der Serotypen Poliovirus 1, 2 und 3. Ausserdem gibt es heute auch in der Umwelt die attenuierten (abgeschwächten) Viren, die als Impfstoff bei der Schluckimpfung verwendet werden. Alle Polioviren haben eine hohe Mutationsrate ${ }^{9}$. Es wäre also denkbar, dass früher in der Natur Poliovirusstämme von der Art der heutigen Impfstämme vorherrschten, die gelegentlich zu aggressiven Varianten mutierten, ohne dass diese Varianten sich auf die Dauer etablieren konnten. Fast alle Menschen hätten sich im Kindesalter mit diesen harmlosen Polioviren infiziert und wären dann gegen die aggressiven Varianten immun gewesen. In der Dritten Welt könnte dieser Zustand bis etwa 1950 gedauert haben, in Nordamerika und Europa aber nur bis zum Anfang des Jahrhunderts. Um diese Zeit hätten dort neu eingeführte Methoden der Desinfektion des Trinkund Abwassers (zur Verhütung der Cholera) dazu geführt, dass aggressivere Virusstämme die älteren, harmlosen Stämme verdrängt haben.

Weiter kann man annehmen, dass die neuen Stämme während des 2. Weltkriegs durch Truppen nach Nordafrika und Ostasien verschleppt worden sind. Da im Verlauf der Kämpfe die sanitären Gewohnheiten der deutschen, englischen und amerikanischen Soldaten natürlich nicht beibehalten wurden, kam es zu den oben erwähnten Epidemien unter Soldaten und wahrscheinlich auch zu ersten Infektionen der Einheimischen mit dem neuen Virus. Dieser Prozess könnte sich nach dem 2. Weltkrieg fortgesetzt haben, als amerikanische Truppen in China und Korea, englische in Indien und Afrika, französische Truppen in Indochina eingesetzt wurden. In Israel dürfte die Einwanderung von Juden aus Europa der entscheidende Faktor gewesen sein.

Die Theorie der Altersabhängigkeit nimmt dagegen an, dass im Laufe der letzten 100 Jahre keine genetische Veränderung der natürlichen Polioviren zu grösserer Virulenz stattgefunden habe. Es habe sich lediglich das mittlere Infektionsalter nach oben verschoben. In unhygienischen Verhältnissen, wie sie früher vorherrschten, infizierten sich fast alle Kinder schon im ersten oder zweiten Lebensjahr. Da somit fast alle Menschen schon mit 3 Jahren ihre 
Infektion hinter sich hatten und dadurch immun waren, wurde die kritische Masse für den Ausbruch einer Epidemie nicht erreicht.

Dieser Abschnitt in der Entwicklungsgeschichte der Poliomyelitis kann im Rahmen der Theorie als die endemische Phase bezeichnet werden. Sie wird, so die Theorie, abgelöst von der epidemischen Phase. Mit zunehmender Höhe des hygienischen und sanitären Standards hat sich nämlich zuerst in den Industrieländern das mittlere Infektionsalter nach oben verschoben. Dadurch hat sich der Anteil der suszeptiblen (nichtimmunen) Personen vorübergehend auf das Doppelte oder Dreifache erhöht, und als Folge davon kam es bei Hinzutreten auslösender Momente zu Epidemien. Solche Momente sind z. B. das Baden in öffentlichen Bädern im Sommer oder der Feldeinsatz von grossen Armeekontingenten mit der dadurch bedingten Lockerung des sanitären Standards. Der historische Übergang von der endemischen zur epidemischen Phase vollzieht sich nach dieser Theorie in jedem Land autonom und im Einklang mit dessen soziokultureller Entwicklung.

Weiter soll die Verschiebung des Infektionsalters schon ausreichen, um das Hochschnellen der Erkrankungszahlen zu erklären. Die Wahrscheinlichkeit, dass eine nichtimmune Person nach der Infektion Symptome entwickelt, nimmt in der Tat mit dem Alter zu, ebenso die Schwere der Symptome.

Die Anhänger dieser Theorie sind nun auch davon überzeugt, dass es die gleichen Polioviren, die für die grossen Epidemien in Europa und Nordamerika verantwortlich waren, auch in der Dritten Welt schon immer gegeben habe. Die dramatische Zunahme der gemeldeten Krankheitsfälle nach dem 2. Weltkrieg führen sie auf die additive Wirkung von zwei Faktoren zurück: Verbesserung des Meldewesens und Verbesserung der sanitär-hygienischen Bedingungen, worauf im Sinne der Theorie der Altersabhängigkeit ja automatisch eine Erhöhung des Infektionsalters und eine Zunahme der Erkrankungen folgen soll.

\section{Die Diskussion in den Jahren 1943-60}

\subsection{Beobachtungen und Aussagen der Militärärzte}

Als im 2. Weltkrieg anglo-amerikanische Truppen in Ägypten, Indien und Ostasien von Polio-Epidemien betroffen wurden, tauchte die Frage auf, ob die Soldaten durch ein einheimisches «Reservoir» von Polioviren infiziert 
worden waren oder ob sie die Viren aus der Heimat mitgebracht hatten. In diesem Zusammenhang fragte man natürlich auch nach der Inzidenz der klinischen Poliomyelitis in der einheimischen Bevölkerung. In den ersten Berichten aus Ägypten und Indien überwog die Meinung, dass diese sehr niedrig gewesen sei ${ }^{10,11}$. Dieser Befund schien auf einen Infektionsimport hinzudeuten. Entsprechend äusserte sich im Dezember 1944 der amerikanische Sanitätsoffizier Henry M. Thomas in einem Brief von der Philippineninsel Leyte an seinen Vorgesetzten: «Certain facts seem fairly sure, however, and these point to the introduction of the reservoir into Leyte with the troops» ${ }^{6}$.

In den folgenden Jahren tauchte aber schon gelegentlich die These auf, dass klinische Fälle von Poliomyelitis auch früher in Afrika und Asien recht häufig gewesen, aber weder von einheimischen Meldesystemen noch von ausländischen Beobachtern registriert worden seien. Hierzu bemerkt jedoch damals, 1947, Albert B.Sabin in seiner Arbeit über die Epidemiologie der Poliomyelitis: «While there are justifiable doubts regarding the adequacy of diagnosis and reporting in former years, the bulk of the evidence suggests that the epidemic outbursts, of the type which have occurred especially in the United States and a few other countries in the past thirty to forty years, are events that could not readily have been dismissed in the past. As a matter of fact it has been pointed out, and in my opinion correctly, that more adequate diagnosis and reporting are the effect rather than the cause of increased incidence». Er geht dann auf die Situation in China ein und gibt zu bedenken, dass in Peking, Tientsin und Shanghai «only rare sporadic cases have been recorded so far, despite the presence now for many years of excellent western-trained physicians who could not have missed such outbreaks in the native population if they had occurred»?

In der gleichen Arbeit berichtet Sabin als Augenzeuge von einer PolioEpidemie, die im Sommer 1947 in einer Einheit von US-Marines in der Nähe von Tientsin in Nordchina ausgebrochen war. Wie sah es gleichzeitig in der Umgebung aus? «There was no evidence of an outbreak of Poliomyelitis in the native population at the time, and Dr. Grice, a British physician in practice in Tientsin for 25 years, informed me that while he not unfrequently saw paralytic poliomyelitis in children in the foreign colony he rarely saw the disease among the Chinese ${ }^{7}$. Zur Frage, wie die Epidemie in der US-Einheit entstanden sein könnte, macht Sabin keine Aussage. Aber seine Überzeugung, dass die niedrige Inzidenz in der einheimischen Bevölkerung kein Artefakt ist, tritt klar hervor. 
John R. Paul, der während des 2. Weltkriegs in Ägypten als Militärarzt tätig war, hatte schon 1949 eine andere Auffassung formuliert: «It is likely that clinical Poliomyelitis may be much more prevalent in some areas, than has previously thought to be the case ${ }^{12}$. Was hier noch vorsichtig als Vermutung geäussert wird, wurde später zum Dogma.

\subsection{Virologische Untersuchungen in Afrika}

Um die von Vertretern der Theorie der Altersabhängigkeit vorgebrachte These, dass Poliovirus 1, 2 und 3 schon länger in Afrika und Asien endemisch gewesen sei, mit virologischen Untersuchungen abzustützen, hätte man möglichst früh versuchen müssen, diese Viren auch an Orten nachzuweisen, die bis dahin von Weissen nicht besiedelt und nur selten oder nie besucht worden waren. Ausserdem hätte man entsprechende Studien so anlegen müssen, dass auch konkurrierende Viren mit gleicher Wahrscheinlichkeit hätten gefunden werden können.

Tab. 1. In der Südafrikanischen Union (SAU), Südrhodesien (Rho), Kenia und benachbarten Ländern in den Jahren 1947- 53 isolierte Polioviren (Quelle: [3])

$\begin{array}{lllll}\text { Anzahl } & \text { Jahr } & \text { Ort } & \text { Ursprung } & \text { Serotyp } \\ 1 & 1947 & \text { Swasiland } & \text { symptomlose Infektion } & 1 \\ 1 & 1948 & \text { Johannesburg } & \text { Abwasser } & 1 \\ 1 & 1949 & \text { Nairobi } & \text { Abwasser } \\ 2 & 1950 & \text { Nyassaland } & \text { paralytischer Fall, Stuhl } & 1 \\ 1 & 1951 & \text { Boksburg/SAU } & \text { paralytischer Fall, Stuhl } & 1 \\ 3 & 1951 & \text { Salisbury/Rho } & \text { paralytischer Fall, Stuhl } & 1 \\ 1 & 1951 & \text { Salisbury/Rho } & \text { Kontakt zweier tödlicher Fälle } & 1 \\ 3 & 1952 & \text { Salisbury/Rho } & \text { paralytischer Fall, Stuhl } & 1 \\ 1 & 1952 & \text { Salisbury/Rho } & \text { paralytischer Fall, Stuhl } & 2 \\ 1 & 1952 & \text { Johannesburg } & \text { paralytischer Fall, Stuhl } & 1 \\ 1 & 1952 & \text { Germiston/SAU } & \text { symptomlose Infektion } & 1 \\ 1 & 1952 & \text { Kapstadt } & \text { tödlicher Fall, ZNS } & 2 \\ 2 & 1953 & \text { Boksburg/SAU } & \text { paralytischer Fall, Stuhl } & 1 \\ 1 & 1953 & \text { Evaton/SAU } & \text { paralytischer Fall, Stuhl } & 1 \\ 1 & 1953 & \text { Johannesburg } & \text { Kontaktperson, Stuhl } & 3 \\ 2 & 1953 & \text { Johannesburg } & \text { paralytischer Fall, Stuhl } & 3 \\ 1 & 1953 & \text { Johannesburg } & \text { paralytischer Fall, Stuhl } & 1 \\ 1 & 1953 & \text { East London/SAU } & \text { paralytischer Fall, Stuhl } & 2 \\ 2 & 1953 & \text { Johannesburg } & \text { paralytischer Fall, Stuhl } & 1 \\ 2 & 1953 & \text { Johannesburg } & \text { paralytischer Fall, Stuhl } & 3\end{array}$




$\begin{array}{lllll}\text { Anzahl } & \text { Jahr } & \text { Ort } & \text { Ursprung } & \text { Serotyp } \\ 1 & 1953 & \text { Johannesburg } & \text { Kontaktperson, Stuhl } & 1 \\ 1 & 1953 & \text { Johannesburg } & \text { Kontaktperson, Stuhl } & 3 \\ 1 & 1953 & \text { Johannesburg } & \text { paralytischer Fall, Stuhl } & 2 \\ 2 & 1953 & \text { Salisbury/Rho } & \text { paralytischer Fall, Stuhl } & 2 \\ 1 & 1953 & \text { Kapstadt } & \text { paralytischer Fall, Stuhl } & 3\end{array}$

Die in den Jahren 1947-53 in Afrika durchgeführten Studien, über die in der WHO-Monographie berichtet wird (Tab. 1), genügen diesen Kriterien nicht. Aus insgesamt 36 Personen wurde Poliovirus 1, 2 oder 3 isoliert. Unter den Herkunftsländern finden sich jedoch nur Länder mit einer bedeutenden weissen Minderheit: die Südafrikanische Union, die ehemalige Föderation Rhodesien-Nyassaland (250 000 Weisse, 1956), Kenia (30000 Weisse, 1948) und das kleine, auf drei Seiten von der Südafrikanischen Union eingeschlossene Swaziland, damals ein britisches Protektorat. In 32 Fällen wurden die Proben von Personen genommen, die schwer oder tödlich erkrankt waren oder mit Kranken Kontakt hatten. In nur 2 Fällen war der Ursprung eine symptomlose Infektion, und in 2 Fällen das Abwasser einer Grossstadt. Man hat also in 32 Fällen die Proben dort genommen, wo man die aggressiven Stämme finden musste. Welche andern Viren im Abwasser von Johannesburg und Nairobi präsent waren, wird nicht gesagt.

\subsection{Die WHO-Monographie}

Die Monographie «Poliomyelitis», die 1955 in englischer und französischer Fassung als Nr.26 in der Reihe der WHO-Monographien erschienen ist, enthält zwei Artikel, die in diesem Zusammenhang besonders relevant sind.

4.3.1. Die Poliomyelitis in den ungenügend entwickelten Regionen, von James H.S. Gear ${ }^{3}$

Der Verfasser war Director of Research am Laboratorium der Polimyelitis Research Foundation des Südafrikanischen Instituts für Medizinische Forschung in Johannesburg. Sein Beitrag enthält einen Überblick über die gemeldeten Poliofälle in allen «ungenügend entwickelten» Ländern, in denen überhaupt gemeldet wurde. Ferner wird über die bereits genannten virologischen Studien in Südafrika, Rhodesien und Kenia berichtet. Gear macht jedoch nicht, wie andere nach ihm, den Fehler, aus den Ergebnissen dieser Studien auf die Absenz von schwach virulenten Virusstämmen in 
Afrika zu schliessen. Gleichwohl ist er davon überzeugt, dass virulente Stämme in Afrika schon mindestens seit einigen Generationen präsent gewesen sind, und erklärt die auffallend niedrige Inzidenz von paralytischer Polio bei den Afrikanern mit einem Selektionseffekt auf der Seite des menschlichen Wirts: da die Stammesbräuche die Fortpflanzung von Lahmen nicht zuliessen, seien menschliche Genotypen, die zu paralytischem Verlauf der Polio-Infektion prädestinierten, in Afrika sehr selten geworden. Durch die viel höhere Paralyse-Inzidenz, die heute in Afrika beobachtet wird, ist diese Theorie widerlegt.

4.3.2. Die Immunität gegen Poliomyelitis in Beziehung zur Impfung, von A. B. Sabin ${ }^{3}$

Dieser Beitrag zeigt, dass die Idee der Impfung mit attenuierten Viren in Sabins Denken nicht zu trennen war von der Vorstellung, dass es auch in der Natur immunisierende Polioviren von schwacher Virulenz gebe oder einmal gegeben habe. Nachdem er gegen drei verschiedene Varianten der Theorie der Altersabhängigkeit (Schutz des Säuglings a) durch plazentare Antikörper, b) durch Antikörper in der Muttermilch, c) allmählicher Anstieg der Empfindlichkeit mit dem Alter) empirisches Material und stichhaltige Argumente vorgebracht hat, formuliert er seine eigene Erklärung des Paradoxons der niedrigen Paralyse-Inzidenz in Ländern mit primitiver Hygiene: «Ich habe anderswo epidemiologische Beweise diskutiert, die anzeigen, dass eine weit verbreitete Immunität aus der Infektion mit schwach virulenten Stämmen resultieren kann» ${ }^{13 a}$.

Als Beispiel führt Sabin die Epidemie auf der Insel Malta an, die dort im November 1942 nach langer Belagerung und schwerer Bombardierung durch italienische und deutsche Streitkräfte ausbrach. Nur maltesische Kinder bis zu 5 Jahren und britische Soldaten erkrankten, während die älteren Einwohner Maltas verschont blieben. Nun ist belegt, dass es in den 40 Jahren davor keine Epidemie auf der Insel gegeben hatte. Die diesbezüglichen Angaben sind glaubwürdig, denn die britische Kolonie Malta hatte schon im 19. Jahrhundert ein gut organisiertes Gesundheitswesen ${ }^{14}$. Sabin zieht aus den genannten Fakten den Schluss, dass die ältere maltesische Bevölkerung durch schwach virulente Polioviren immunisiert war und dass im Jahre 1942 ein sehr virulenter Stamm auf der Insel aufgetaucht sein muss. Die Möglichkeit, dass dieser virulente Stamm mit den ersten britischen Schiffen, die 
nach der Belagerung in Malta eintrafen, gekommen sein könnte, übergeht er jedoch stillschweigend und spricht stattdessen von der «Tatsache, dass solche Stämme periodisch in den fraglichen Gegenden angetroffen werden». Hier endet die logische Beweisführung abrupt, denn diese «Tatsache» ist für Sabin durch die Polio-Epidemien unter anglo-amerikanischen Soldaten in Afrika und Asien hinreichend bewiesen. Dass in manchen Gebieten der Erde avirulente und virulente Stämme sich auf natürliche Weise abwechseln, kann zwar nicht ganz von der Hand gewiesen werden, aber wie wäre es dann zu erklären, dass die virulenten Stämme an verschiedenen Orten immer gerade dann aufgetaucht sind, als britische oder amerikanische Truppen anwesend waren?

\subsection{Sabins letztes Wort}

In den späten 50er Jahren gelang Sabin der entscheidende Durchbruch zur oralen Polio-Vakzine, und 1960 wurde der nach ihm benannte Impfstoff in den Vereinigten Staaten zugelassen. Zur gleichen Zeit gab das Medical Department der US Army ein umfangreiches Werk über Präventivmedizin im 2. Weltkrieg heraus, für das Sabin den Artikel über Poliomyelitis schrieb $^{6}$.

Sabins Beitrag beginnt mit einer Historical Note, die der Zeit vor dem 2. Weltkrieg gewidmet ist. Es fällt auf, wie hier ein Ausbruch auf den Philippinen, die damals eine amerikanische Kolonie waren, kommentiert wird. Im Jahre 1934, wurden 17 Polio-Patienten, sämtlich Militärpersonen und Familienangehörige (dependents) von solchen, in das Sternberg General Hospital in Manila aufgenommen. «None of them were recent arrivals from the Unites States ... Aside from the two cases which occurred in the medical officers' families, there was no known direct or indirect contact between the cases». Anscheinend sollen diese Bemerkungen die These stützen, dass die Viren aus einem Reservoir auf den Philippinen stammten.

Weiter wird erwähnt, dass ausser diesen 17 Fällen aus dem ganzen Archipel nur 12 weitere Fälle im gleichen Jahr gemeldet wurden, darunter 4 amerikanische Zivilisten, 1 Mestize und 7 Philippinos - ein erstaunliches Verhältnis angesichts des minimalen Bevölkerungsanteils der Amerikaner. Für den Sanitätsoffizier Hillman, aus dessen Bericht Sabin zitiert, blieb dies «a mystery». Sabin fährt fort : «With the more limited knowledge ... in 1934, Colonel Hillmann did not even consider the possibility that inapparently 
infected Filipino domestic servants might have been responsible for bringing the infection to the families of the American officers and the civilians». Dies scheint Sabins eigene Erklärung im Jahre 1960 gewesen zu sein. Die Möglichkeit, dass symptomlos infizierte Amerikaner vom Kontinent die Epidemie unter ihren Landsleuten ausgelöst, und dass diese dann ihr einheimisches Hauspersonal infiziert haben könnten, wird schon gar nicht mehr diskutiert.

Hillmann hatte vermutet, die Infektionen bei Amerikanern könnten durch importierte Nahrungsmittel aus den USA entstanden sein. Dazu Sabin: «This speculation is of interest because, during World War II, there were also those who initially were inclined to attribute the unexpected number of cases of Poliomyelitis among American personnel in the Philippine Islands to importation of the virus from the United States.»

Im Hauptteil seiner Arbeit erörtert Sabin die Polio-Ausbrüche in Truppeneinheiten im Mittleren Osten und auf den Philippinen während des 2. Weltkrieges. Die Epidemie auf Malta, die er fünf Jahre früher ausführlich erörtert hatte, kommt nicht wieder zur Sprache, denn er beschränkt sich hier, wohl mit Rücksicht auf den Herausgeber des Werkes, auf die USTruppen. Um die These des Infektionstransfers von den USA nach den Philippinen zu widerlegen, führt Sabin die folgenden Argumente ins Feld:

1. Die Invasionstruppen waren im Mittel länger als 1 Monat auf See, ohne dass ein einziger Fall von Poliomyelitis auftrat.

2. Der erste Fall erschien erst 16 Tage nach Beginn der Invasion.

3. Die Fälle traten in verschiedenen Einheiten auf, die keinen Kontakt miteinander hatten.

4. Als diese Truppen in Neuguinea und auf anderen Inseln im Südwestpazifik standen, wo sie keinen Kontakt mit den Einheimischen hatten, gab es keine Fälle von Poliomyelitis.

5. Auch in den Jahren 1945/46 war die Poliomyelitis unter den US-Truppen auf den Philippinen wesentlich häufiger als in den Einheiten in den USA.

Zum Schluss benennt Sabin die Faktoren, die eine Übertragung der Infektion von Einwohnern der Philippinen auf US-Soldaten begünstigt hätten, ohne sich dessen bewusst zu werden, dass die gleichen Faktoren natürlich auch die Übertragung zwischen Soldaten im Feld und von Soldaten auf Zivilpersonen begünstigen konnten: «One cannot disregard the various routes by which human feces, potentially infected with the virus, may reach 
susceptible individuals ... Due to the lack of latrines, the heavy fly population, and the civilian habit of defecating on the ground, it was not surprising that the troops stationed in the midst of densely populated regions exhibited a high incidence of those infections which are found in the human feces». Um die Schwäche dieser Argumentation aufzudecken, genügt der Hinweis, dass Latrinenbau wohl nicht die erste Aktivität der Invasionstruppen gewesen ist.

\section{Zu den Argumenten im einzelnen:}

ad 1. Bei der Invasion der Philippinen waren auch Einheiten beteiligt, die vorher noch nicht im westlichen Pazifik stationiert waren. Eine Einheit wurde, wie Sabin erwähnt, von Hawaii herangeführt, und sicher kam auch Verstärkung direkt vom Kontinent (insofern ist das «these» in Argument 4 irreführend). Die 7000 Meilen von der amerikanischen Westküste bis zu den Philippinen konnte ein Truppentransporter wohl in weniger als 2 Monaten zurücklegen. Da mehr als $99 \%$, nach manchen Schätzungen 99,9\% der Polio-Infektionen symptomlos verlaufen, ist die Wahrscheinlichkeit, dass ein Cluster von 10 bis 20 Infektionen auf einem Schiff bemerkt wurde, so klein, dass die Überfahrt von infizierten Soldaten nach anerkannten Regeln der Statistik nicht ausgeschlossen werden kann. Da die Viren bis 4 Monate lang ausgeschieden werden, können diese Soldaten nach der Landung, als die Einhaltung sanitärer Standards vorübergehend unmöglich war, Tausende ihrer Kameraden angesteckt haben.

ad 2. Da die meisten Glieder einer Infektionskette unerkannt bleiben, muss eine importierte Polio-Epidemie nicht unmittelbar nach der Landung zu klinischen Fällen führen.

ad 3. In die verschiedenen Einheiten kann das Virus jeweils durch Rekruten vom amerikanischen Kontinent Eingang gefunden haben.

ad 4. Die Landung in Neuguinea erfolgte bereits im Mai, die auf Leyte dagegen im Oktober ${ }^{15}$. Dies ist entscheidend, denn die Polio-Epidemien in den Vereinigten Staaten waren immer saisonabhängig, mit dem Höhepunkt im Sommer oder Frühherbst. Die Tatsache, dass nur auf den Philippinen, wo die Soldaten Kontakt mit der Bevölkerung hatten, Poliomyelitis in der Armee auftrat, ist also kein stichhaltiges Argument für die These, dass die Soldaten sich durch diesen Kontakt infiziert haben.

ad 5. Auch nach dem Ende der Kämpfe können die bereits genannten Umweltfaktoren eine Erklärung dafür geben, dass die Soldaten auf den Philippinen stärker betroffen waren als die in Amerika. Damit ist auch das Argument 5 entkräftet. 


\section{Die Etablierung eines Dogmas}

Die Theorie der Altersabhängigkeit wurde bereits von anderen Autoren als Dogma bezeichnet ${ }^{16}$. Nachdem Sabin mit seiner ganzen Autorität als Schöpfer des oralen Impfstoffs seinen Widerspruch zurückgezogen hatte, stand der Ausbreitung des Dogmas nichts mehr im Wege. Die einzige bisher erschienene «Geschichte der Poliomyelitis» von John R. Paul liegt ganz auf der Linie des Dogmas ${ }^{17}$. Paul erwähnt auch die Polio-Epidemien bei der Invasion der Philippinen und führt, wie zu erwarten, Sabin als Kronzeugen an: «Fifteen years later Sabin gave five reasons why colonel Thomas' interpretation was incorrect and presented evidence that favored the local native population of Leyte as the actual reservoir of the virus» ${ }^{17 a}$. Sabins fünf Gründe, die wir vorhin diskutiert haben, werden jedoch von Paul dem Leser vorenthalten!

Die früher sehr niedrige Paralyse-Inzidenz in Afrika und Asien ist für Paul blosser Schein. Er zitiert einen ägyptischen Kinderarzt, der im Sommer 1943 gesagt haben soll: «Poliomyelitis is not rare in Egypt. It is never epidemic always sporadic. It is a disease of poorer classes. The cases are as a rule mild, involving only one limb - rarely a fatality ${ }^{17 \mathrm{~b}}$. Bezeichnenderweise wird aber die Epidemie auf Malta, die in Sabins WHO-Artikel von 1955 eine Schlüsselrolle gespielt hat, mit nur 5 Zeilen abgehandelt. Auch die Frage, warum die Erkrankung der ägyptischen Kinder in der Regel mild verlief, wird nicht gestellt. Hingegen glaubt Paul, trotz der ins Auge springenden epidemiologischen Besonderheiten, die Poliomyelitis in eine allgemeine Kategorie von Krankheiten, die aus dem Schmutz entstehen, einordnen zu können:

«Previously it had been observed by military medical men that when troops from a developed country occupy an area inhabited by a population with insanitary habits where the environmental sanitation is primitive, military personnel are likely to suffer from a variety of infections common to the natives of that area, particularly during the first few months after their arrival. These include all kinds of dysenteries, hepatitis, and other acute viral and parasitic infections, even typhoid fever. During World War II poliomyelitis was added to this group. It had suddenly become a disease with which the armed forces had to reckon» ${ }^{17 c}$.

\section{Voraussetzungen für eine wissenschaftliche Lösung des Problems}

Aus den obigen Ausführungen dürfte die ungenügende epidemiologische und virologische Begründung des «Dogmas» hinreichend deutlich geworden 
sein. Dass es zur Herrschaft kommen konnte, hängt vielleicht mit dem Einfluss der militärischen Hierarchie auf führende Forscher der USA in den 50er Jahren zusammen - der entscheidende Artikel von Sabin wurde ja im Auftrag der US Army geschrieben. Dass es 34 Jahre später immer noch herrscht, hat freilich andere Gründe. Der wichtigste Grund ist wohl die weit verbreitete Tendenz, Publiziertes ungeprüft zu übernehmen und zu zitieren.

Für die Mutationstheorie gibt es bisher keine empirischen Beweise, weil niemand es ernsthaft unternommen hat, Beweise zu sammeln. Eine entscheidende Frage ist die folgende:

War die niedrige gemeldete Paralyse-Inzidenz in Afrika und Asien vor 1947 ein Meldeartefakt oder entsprach sie den Tatsachen?

Die Antwort könnte leicht gefunden werden, denn die Jahrgänge, die vor 1947 als Kinder der Infektion durch Polioviren ausgesetzt waren, sind heute 48 Jahre und älter, d.h. trotz der höheren Sterblichkeit in den fraglichen Ländern sind noch so viele am Leben, dass man den Prozentsatz von Gelähmten unter den noch Lebenden eines Jahrgangs bestimmen und von da auf den gesamten Jahrgang hochrechnen könnte. Dass hierbei noch einige methodische Probleme zu lösen sind (z. B. grössere Sterblichkeit der Gelähmten, Lähmungen mit anderen Ursachen), ist klar, aber grundsätzlich ist der Weg gangbar.

\section{Epilog}

Im Jahre 1981 ist Sabin zu den Thesen, die er in der Nachkriegszeit vertreten hatte, zurückgekehrt: «The increasing numbers of epidemics in the tropics since 1945 occurred in populations with high infant mortality rates and poor living conditions and were generally associated with development projects that brought together thousands of families from isolated villages. Because almost all of the paralytic cases in these epidemics still occurred in children under two or three years of age, it appeared that the paralytic disease was due to the introduction and extensive dissemination of highly neurovirulent strains of polioviruses to which the older children were already immune as a result of their previous exposure to polioviruses of low neurovirulence in their native villages» ${ }^{18}$. Diese überaus deutliche Absage an das «Dogma» befindet sich jedoch in einer Publikation, die in der Hauptsache einem ganz anderen Thema - der Rolle von neu entdeckten Enteroviren als Ursache von Lähmungen - gewidmet ist, und wurde deshalb kaum zur Kenntnis genommen. 


\section{Literatur}

1 Heine J., Spinale Kinderlähmung. Stuttgart 1860.

2 Sabin A.B., Paralytic consequences of Poliomyelitis infection in different parts of the world and in different population groups. Amer. J. Publ. Health 41, 1215-1230, 1951.

3 Gear J., La poliomyélite dans les régions insuffisamment développées. In: La poliomyélite, OMS (WHO), Monographie 26, Genève 1955.

4 Melnick J., Chapt. 33 in: Virology (ed. B. N. Fields), New York 1985.

5 Centers for Disease Control (Atlanta/USA), Oct. 1989

6 Sabin A. B., Poliomyelitis. In: Preventive Medicine during World War II, vol. 5. Office of the Surgeon General, Dept. of the Army, Washington 1960, pp. 367-400.

7 Sabin A. B., The epidemiology of Poliomyelitis. Problems at home and among the Armed Forces abroad. J. Amer. Med. Ass. 134, 749-56, 1947.

8 Knolle H., Übertragung der Poliomyelitis durch Trinkwasser und das Problem der Ausrottung, in Vorbereitung.

9 Bienz K. et al., Swiss feasability study for the surveillance of poliovirus in the environment, Universität Basel 1991.

10 Illingworth 1945, zitiert in Ref. 12.

11 Paul, Havens, van Rooyen 1944, zitiert in Ref. 12.

12 Paul J. R., Poliomyelitis attack rates in American troops. Amer. J. Hygiene 49, 57-62, 1949.

13 Sabin A.B., L'immunité dans la poliomyélite en rapport avec la vaccination. In: La poliomyélite, OMS (WHO), Monographie 26, Genève 1955.

13a loc. cit., p. 326 .

14 Cassar P., Medical History of Malta, London 1964.

15 Encycl. Britannica (1961), Artikel World War II.

16 Nathanson N. \& Martin J., The epidemiology of Poliomyelitis: enigmas surrounding its appearance, epidemicity and disappearance. Amer. J.Epid. 110, 672-92, 1979.

17 Paul J. R., A History of Poliomyelitis, Yale Univ. Press 1971.

17a loc. cit., p. 354 .

17b loc. cit., p. 353 .

17e loc. cit., p. 355 .

18 Sabin A.B., Paralytic Poliomyelitis: Old dogmas and new perspectives. Rev. Infect. Diseases 3, 543-564, 1981.

PD Dr. Helmut Knolle

Institut für Sozial- und Präventivmedizin der Universität Bern

Finkenhubelweg 11

CH-3012 Bern 